CHLAMYDIA

\title{
Discordance between trends in chlamydia notifications and hospital admission rates for chlamydia related diseases in New South Wales, Australia
}

\section{Y Chen, C K Fairley, B Donovan}

Sex Transm Infect 2005;81:318-322. doi: 10.1136/sti.2004.012807

See end of article for authors' affiliations

...................

Correspondence to: Dr Marcus Chen, Melbourne Sexual Health Centre, 580 Swanston Street, Carlton, Victoria, Australia, 3053; mchen@ mshc.org.au

Accepted for publication 24 January 2005 es for chlamydia have increased fourfold since the early $1990 \mathrm{~s}$. Background: In Australia, notification rates for chlamydia have increased fourfold since the early 1990 s.
An increasing incidence of genital Chlamydia trachomatis infection would be expected to lead to a rise in An increasing incidence of genital Chlamydia
the incidence of chlamydia related diseases.

Objectives: To determine trends in hospital admission rates for pelvic inflammatory disease (PID), ectopic pregnancy, and epididymo-orchitis in New South Wales.

Methods: Age specific admission rates from 1992 to 2001 were ascertained using inpatient data from all hospitals within the state.

Results: Among women aged 15-34 years, hospital admission rates for PID fell from 165 per 100000 population in 1992 to 64 per 100000 population in 2001 (p for trend $<0.0001$ ). 15\% of PID cases in women aged 15-24 years were chronic, compared with $32 \%$ in those aged $25-34$ years, and $46 \%$ in the 35-44 year age group. The incidence of ectopic pregnancy and admission rates for epididymo-orchitis remained constant.

Conclusions: On a population level, trends in the incidence of chlamydia related diseases do not necessarily parallel those of reported chlamydia rates and ecological associations between the two need to be interpreted with caution.
$\mathrm{L}$ ower genital tract infection with Chlamydia trachomatis and Neisseria gonorrhoeae can lead to pelvic inflammatory - disease (PID) in women and epididymo-orchitis in men. In turn, PID is commonly followed by ectopic pregnancy, tubal factor infertility, or chronic pelvic pain, resulting in substantial reproductive morbidity. ${ }^{1}$ In randomised controlled trials, selective chlamydia testing and treatment of women has been associated with decreases in the incidence of PID. ${ }^{23}$ In men under the age of 35 years, epididymitis is usually the result of $C$ trachomatis infection, while urinary tract pathogens are generally responsible for epididymitis in older men. ${ }^{45}$

In Australia, notification rates for chlamydia have increased fourfold since the early 1990s, with the greatest rises seen among adolescents and young adults (fig 1 ). ${ }^{6}$ In New South Wales, Australia's most populous state, legislation was introduced in 1998 that made the reporting of $C$ trachomatis by laboratories mandatory. Chlamydia notifications in New South Wales increased in parallel with national rates-from 39 per 100000 population in 1999 to 88 per 100000 in 2002. ${ }^{7}$ The extent that these increases in chlamydia notifications represent a true rise in chlamydia incidence is uncertain: ecological studies suggest the higher notifications rates also reflect increased chlamydia testing. ${ }^{8} 9$

During the 1990s, gonococcal notifications among women in New South Wales remained at historically low levels, while increases among men were largely restricted to those who were homosexually active. Throughout the 1990s, annual gonorrhoea notifications in women remained below 3.5 per 100000 population. ${ }^{1011}$

Given the rise in chlamydia notifications and the uncertainty over its significance, we sought to establish if the

Abbreviations: HOIST, Health Outcomes Indicator Statistical Toolbox; PID, pelvic inflammatory disease hospital inpatient morbidity from specific chlamydia related complications had also increased. The aim of this study was to determine trends in the incidence of hospital admissions for PID, ectopic pregnancy, and epididymo-orchitis in New South Wales, Australia's most populous state with a population of 6.6 million.

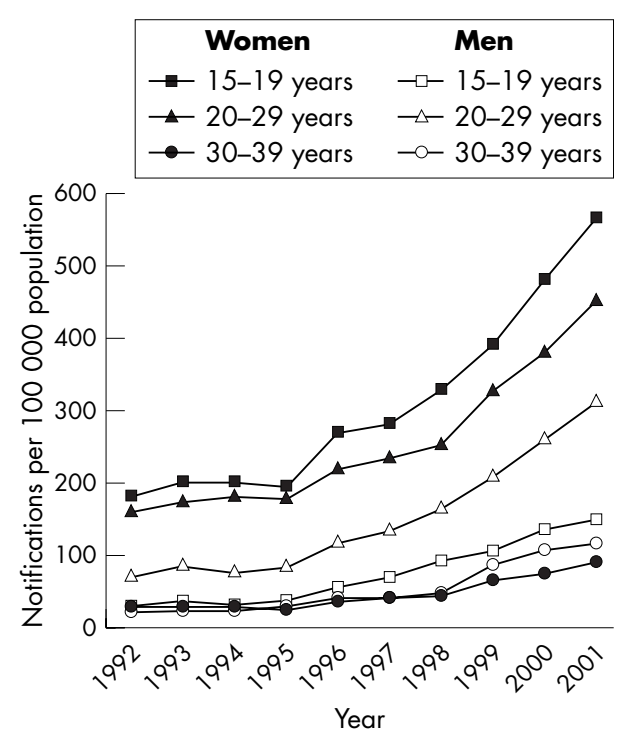

Figure 1 Notification rates for genital Chlamydia trachomatis infection in women and men by age group, Australia, 1992-2001. (Notifications commenced in New South Wales in 1998.) (Source: National Centre in HIV Epidemiology and Clinical Research; www.med.unsw.edu.au/ nchecr//) 


\section{METHODS}

Information on all patients admitted for PID, ectopic pregnancy, or epididymo-orchitis to all New South Wales hospitals_both public and private-between 1992 and 2001 was obtained from the state health department, via the Health Outcomes Indicator Statistical Toolbox (HOIST) ${ }^{12}$ using SAS. ${ }^{13}$ Only cases where PID, ectopic pregnancy, or epididymo-orchitis were indicated as the main reason for admission were included. These diagnoses were identified using concordant ICD9 (numeric) and ICD10 (alphanumeric) codes (table 1$).{ }^{14}$ When these three conditions were recorded as additional diagnoses they were excluded from this study as they may have represented pre-existing or historical conditions. ${ }^{12}$ As all subjects were anonymous, whether individuals underwent repeat admissions during the study period could not be determined. Data on visits to hospital emergency departments that did not result in admission were also excluded.

ICD10 categories A56.1 (chlamydial infection of pelviperitoneum and other genitourinary organs) and K67.0 (chlamydial peritonitis $)^{14}$ were not included as these could not be translated retrospectively into concordant ICD9 categories. These diagnoses were, in any case, rarely used and accounted for less than $0.5 \%$ of cases. For 2001, whether a woman underwent laparoscopy or laparotomy was ascertained by examining details of procedures performed during admission from information available from the HOIST database: this information was not available for previous years.
Age specific hospital admission rates for PID and epididymo-orchitis (cases per 100000 population) were calculated using annual New South Wales population data from the Australian Bureau of Statistics. ${ }^{15}$ The age specific incidence of ectopic pregnancy (cases per 10000 births) was determined based on annual birth rates from the New South Wales Midwives Data Collection. The significance of trends in admission rates over time was measured by the MantelHaenszel $\chi^{2}$ test using SAS. ${ }^{13}$

Data on hospital inpatients were de-identified to protect patient confidentiality. Ethical approval for this study was granted by the human research ethics committee of the University of Sydney.

\section{RESULTS}

The number of hospital admissions per year according to ICD classification is shown in table 1. Admission rates for PID among women aged 15-34 years fell from 165 per 100000 in 1992 to 64 per 100000 in 2001 ( $p$ for trend $<0.0001$ ) (fig 2). The reduction in PID rates for women aged 35-44 years was less pronounced, and overall rates were lower.

In over half of PID cases, whether it was acute or chronic was not specified (fig 2). For the 10 year period, PID of unspecified duration accounted for $55.8 \%$ of diagnoses in the 15-44 year age group. The proportion of women with known chronic PID increased with age. In women aged 15-24 years, $15 \%$ of PID cases during the study period were specified as being chronic, ranging from $13 \%$ to $20 \%$. In those aged

Table 1 Hospital admissions for chlamydia related diseases in men and women aged 15-34 years, New South Wales, 19922001

\begin{tabular}{|c|c|c|c|c|c|c|c|c|c|c|}
\hline Diagnosis (ICD codes)* & 1992 & 1993 & 1994 & 1995 & 1996 & 1997 & 1998 & 1999 & 2000 & 2001 \\
\hline \multicolumn{11}{|l|}{ Acute PID } \\
\hline Acute salpingitis and oophoritis $(614.0, \mathrm{~N} 70.0)$ & 30 & 33 & 16 & 20 & 20 & 17 & 22 & 23 & 15 & 19 \\
\hline $\begin{array}{l}\text { Acute inflammatory diseases of the uterus (615.0, } \\
\text { N71.0) }\end{array}$ & 29 & 37 & 16 & 31 & 16 & 28 & 34 & 38 & 23 & 17 \\
\hline $\begin{array}{l}\text { Acute parametritis and pelvic cellulitis (614.3, } \\
\text { N73.0) }\end{array}$ & 31 & 44 & 50 & 46 & 46 & 29 & 44 & 44 & 54 & 54 \\
\hline $\begin{array}{l}\text { Subtotal } \\
\text { Chronic PID }\end{array}$ & 90 & 114 & 82 & 97 & 82 & 74 & 100 & 105 & 92 & 90 \\
\hline $\begin{array}{l}\text { Chronic salpingitis and oophoritis (614.1, } \\
\text { N70.1) }\end{array}$ & 112 & 86 & 96 & 119 & 89 & 70 & 79 & 95 & 79 & 71 \\
\hline $\begin{array}{l}\text { Chronic inflammatory diseases of the uterus } \\
(615.1, \text { N71.1) }\end{array}$ & 138 & 141 & 95 & 130 & 89 & 97 & 93 & 88 & 66 & 53 \\
\hline $\begin{array}{l}\text { Chronic parametritis and pelvic cellulitis (614.4, } \\
\text { N73.1)† }\end{array}$ & 65 & 66 & 84 & 83 & 44 & 30 & 37 & 37 & 34 & 35 \\
\hline Subtotal & 315 & 293 & 275 & 332 & 222 & 197 & 209 & 220 & 179 & 159 \\
\hline \multicolumn{11}{|l|}{ PID of unspecified duration } \\
\hline $\begin{array}{l}\text { Salpingitis and oophoritis of unspecified duration } \\
(614.2, N 70.9)\end{array}$ & 153 & 120 & 111 & 83 & 61 & 68 & 54 & 60 & 43 & 41 \\
\hline $\begin{array}{l}\text { Inflammatory diseases of the uterus of unspecified } \\
\text { duration }(615.9, \mathrm{~N} 71.9)\end{array}$ & 147 & 134 & 122 & 93 & 92 & 46 & 0 & 0 & 0 & 0 \\
\hline $\begin{array}{l}\text { Parametritis and pelvic cellulitis of unspecified } \\
\text { duration (N73.2) } \dagger\end{array}$ & - & - & - & - & - & 13 & 2 & 4 & 2 & 1 \\
\hline $\begin{array}{l}\text { Inflammatory disease of the ovary, fallopian tube, } \\
\text { pelvic cellular tissue and peritoneum (614.8, } \\
614.9, \text { N73.8, N73.9, N74.4) }\end{array}$ & 824 & 727 & 679 & 562 & 517 & 450 & 472 & 325 & 346 & 301 \\
\hline Subtotal & 1124 & 981 & 912 & 738 & 670 & 577 & 528 & 389 & 391 & 343 \\
\hline Total & 1529 & 1388 & 1269 & 1167 & 974 & 848 & 837 & 714 & 662 & 592 \\
\hline Ectopic pregnancy & & & & & & & & & & \\
\hline Other or unspecified $(633.2,633.8,633.9$, & 166 & $\begin{array}{l}955 \\
189\end{array}$ & $\begin{array}{l}940 \\
161\end{array}$ & $\begin{array}{l}929 \\
141\end{array}$ & $\begin{array}{l}889 \\
149\end{array}$ & $\begin{array}{l}882 \\
152\end{array}$ & $\begin{array}{l}888 \\
145\end{array}$ & $\begin{array}{l}856 \\
174\end{array}$ & $\begin{array}{l}879 \\
168\end{array}$ & $\begin{array}{l}813 \\
182\end{array}$ \\
\hline $\begin{array}{l}\text { Total } \\
\text { Epididymo-orchitis }\end{array}$ & 1219 & 1144 & 1101 & 1070 & 1038 & 1034 & 1033 & 1030 & 1047 & 995 \\
\hline Without abscess $(604.90,604.99$, N45.9) & 304 & 309 & 349 & 347 & 350 & 372 & 280 & 295 & 307 & 280 \\
\hline With abscess (604.0, N45.0) & 13 & 10 & 7 & 6 & 9 & 10 & 9 & 4 & 11 & 7 \\
\hline Total & 317 & 319 & 356 & 353 & 359 & 382 & 289 & 299 & 318 & 287 \\
\hline
\end{tabular}



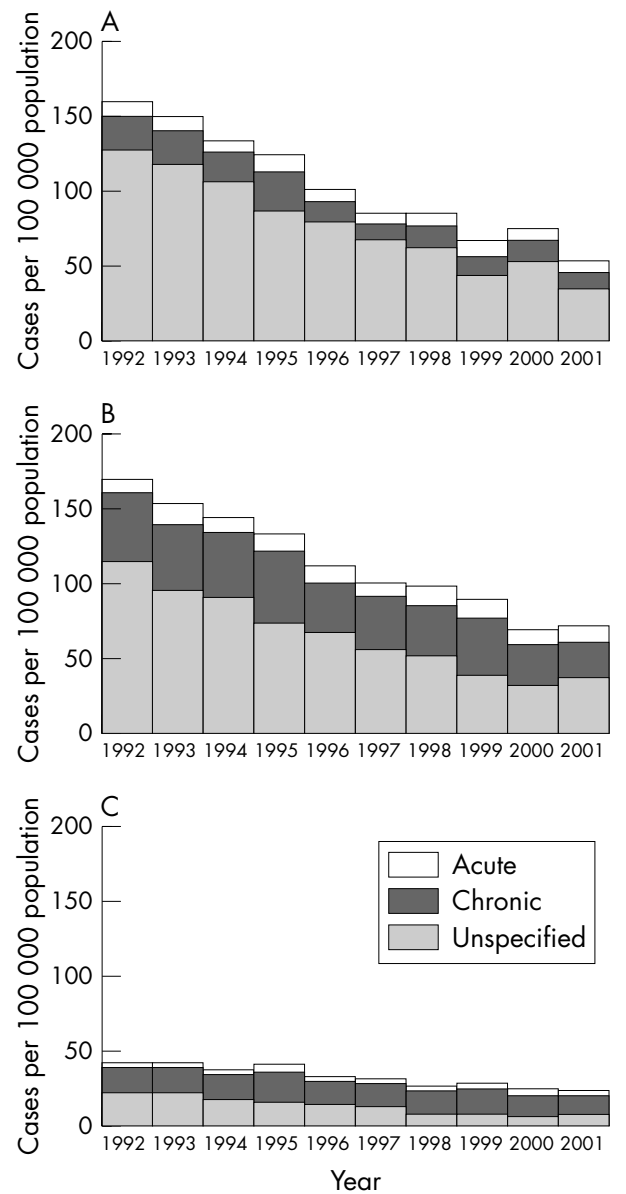

Figure 2 Hospital admission rates for pelvic inflammatory disease in women by diagnosis and age group, New South Wales, 1992-2001. (A) 15-24 years, (B) 25-34 years, (C) 35-44 years. (Source: Inpatients Statistics Collection (HOIST), New South Wales Health Department.)

25-34 years, $32 \%$ of cases overall were chronic, ranging between $27 \%$ and $41 \%$. Among women in the 35-44 year group, chronic PID accounted for $46 \%$ of cases overall, ranging between $38 \%$ and 53\%. In 2001, of the 592 women aged 15-34 years diagnosed with PID, 362 (61.1\%) underwent either laparoscopy or laparotomy.

Over the study period, the incidence of ectopic pregnancy among women aged 15-44 years remained relatively constant

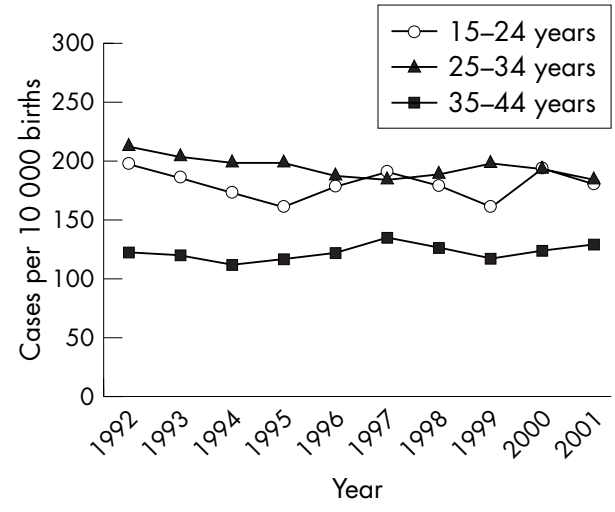

Figure 3 Hospital admission rates for ectopic pregnancy by age group, New South Wales, 1992-2001. (Source: Inpatients Statistics Collection (HOIST), New South Wales Health Department.)

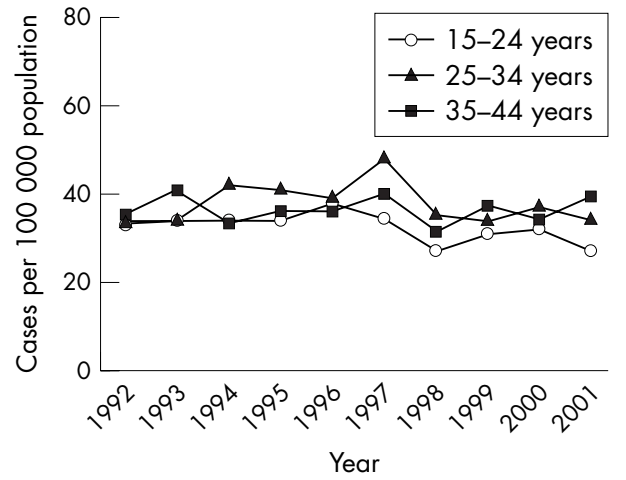

Figure 4 Hospital admission rates for epididymo-orchitis in men by age group, New South Wales, 1992 to 2001. (Source: Inpatients Statistics Collection (HOIST), New South Wales Health Department.)

(fig 3), with 1521 admissions for ectopic pregnancy in 1992 and 1321 in 2001 ( $p$ for trend $=0.06$ ). Eighty five per cent of ectopic pregnancies were specified as being tubal.

Hospital admission rates for epididymo-orchitis in men aged 15-44 years also remained steady (fig 4), with 476 men admitted for epididymo-orchitis in 1992 and 485 in 2001 (p for trend $=0.12$ )

\section{DISCUSSION}

Between 1992 and 2001, hospital admission rates for PID in New South Wales fell progressively, particularly among younger women, while the incidence of ectopic pregnancy and admission rates for epididymo-orchitis were unchanged. These trends occurred despite increasing notification rates for $C$ trachomatis infection. The highest admission rates for PID tended to occur in women aged 20-24 years, and among older women, chronic disease accounted for a greater proportion of PID cases.

Using the HOIST system, we were able to access information on admissions to all hospitals throughout New South Wales. As these hospitals all used the ICD to classify diagnoses, data from different sites were directly comparable. The quality of the data collected is nevertheless dependent upon the standard of coding and the accuracy of clinical diagnoses upon which this is based. The proportion of women with PID who underwent laparoscopy or laparotomy in $2001-61.1 \%$ in those aged 15-34 years-suggests diagnostic accuracy was likely to have been higher than in primary care, where diagnosis usually relies on clinical features only. ${ }^{16}$ PID can be classified using any one of a number of ICD codes, ${ }^{14}$ and coding practices at different hospitals may have varied. ICD classifications are available for both acute and chronic PID, however, most diagnoses of PID in New South Wales were of unspecified duration, a problem also encountered in a US study. ${ }^{17}$ To some extent, the failure to assign PID cases into acute and chronic diagnostic groups probably reflects the absence of a clear clinical distinction between these two conditions.

An important limitation of this study is that it only tells us about cases of PID and epididymitis that were admitted to hospital. Internationally, information on the incidence of PID in primary care is scarce and no data are available for epididymitis. Although some cases of ectopic pregnancy will have been managed on an outpatient basis during the study period, these were probably limited in number. Decreases in PID rates have generally been attributed to concurrent declines in the incidence of chlamydial or gonococcal infection in the community. ${ }^{17}{ }^{18}$ However, because of the ecological design of this study, we are unable to make any 


\section{Key messages}

- In New South Wales, hospital admission rates for pelvic inflammatory disease (PID) fell, while the incidence of ectopic pregnancy and admission rates for epididymo-orchitis remained steady. This occurred despite increases in chlamydia notification rates

- On a population level, trends in the incidence of chlamydia related diseases do not necessarily parallel those of reported chlamydia rates

causal inferences about the relation between chlamydia notification rates and rates of PID.

Progressive falls in hospital admission rates for PID were also seen in Sweden since the $1970{ }^{18}{ }^{18}$ and in the United States $^{16}{ }^{17}$ and Canada ${ }^{19}$ since the early 1980s. In Sweden, decreases in reported rates of chlamydial infection were associated with falls in hospital admission rates for PID and ectopic pregnancy. ${ }^{18} 20$ In New South Wales, however, a divergence between chlamydia notifications and hospital admissions for chlamydia related diseases occurred.

To some degree, the decrease in PID admissions in New South Wales could have reflected reductions in the availability of hospital beds or increased management of PID in the community rather than a true fall in PID incidence. It is worth noting, however, that between 1997-8 and 1999-2000, hospital admission rates for female reproductive disorders in Australia fell by only $1.6 \%{ }^{21}$ while admission rates for PID in women aged 15-34 years in New South Wales fell by $20.7 \%$. In a national representative study of Australian general practices, management rates for PID among younger women fell significantly, with no change in the rates for epididymitis-trends that paralleled those in the present study. ${ }^{22}$ In Australia, PID and epididymitis are much more commonly managed by general practitioners than by hospitals. ${ }^{22}{ }^{23}$

Possible explanations for the apparent fall in PID incidence need to be considered. Although the optimal therapy for PID remains far from certain, it is possible earlier or improved treatment of $C$ trachomatis infection contributed to the reduction in PID. Of note, during the 1990s, the arrival of azithromycin was associated with a marked reduction (from $37 \%$ to $5 \%$ ) of admissions for PID associated with $C$ trachomatis in Darwin. ${ }^{24}$ The use of oral contraceptives has been shown to be protective against symptomatic PID among women infected with $C$ trachomatis ${ }^{1625}$; however, data on patterns of oral contraceptive use in New South Wales are not available. Intrauterine devices are unlikely to have contributed to the fall in PID as less than $0.5 \%$ of women aged $15-$ 49 years in Australia were having intrauterine devices inserted annually by the beginning of the study period. ${ }^{26}$ Although decreasing incidence of gonococcal infection is thought to have contributed to falls in hospital admission rates for PID in Sweden ${ }^{18}$ and the United States, ${ }^{17}$ gonococcal rates among women in New South Wales remained at historically low levels throughout the study period. ${ }^{10}{ }^{11}$

The rise in chlamydia notifications could largely have been the result of increased chlamydia testing. ${ }^{8} 9$ More extensive use of nucleic acid amplification tests may also have contributed ${ }^{27}$ In recent years, chlamydia testing rates have increased steadily throughout Australia, with strong correlations between the intensity of testing and detection rates for C trachomatis. ${ }^{8}{ }^{9}$ Despite this, overall testing rates are still low, with the great majority of infections remaining undetected. ${ }^{8}{ }^{9}$ This makes it difficult to know what the true incidence and prevalence of chlamydia is in the population.
On a population level, trends in the incidence of chlamydia related diseases do not necessarily parallel those of reported chlamydia rates and ecological associations between the two need to be interpreted with caution. Studies to determine the incidence of clinically milder PID and epididymitis in primary care are required.

\section{ACKNOWLEDGEMENTS}

We acknowledge the HOIST system, Epidemiology and Surveillance Branch, New South Wales Health Department, as the source of data for notifications, inpatient statistics, and birth rates. We thank Dr Jeremy McAnulty for his assistance. MC is supported by a National Health and Medical Research Council Medical Postgraduate Scholarship (no 262032).

\section{CONTRIBUTORS}

MC performed data extraction and analysis; all authors were responsible for developing, revising, and rechecking the manuscript.

\section{Authors' affiliations}

M Y Chen, B Donovan, Sydney Sexual Health Centre, Sydney Hospital, and School of Public Health, University of Sydney, Australia

C K Fairley, Melbourne Sexual Health Centre, Alfred Hospital, and School of Population Health, University of Melbourne, Australia

Conflict of interest: none identified.

\section{REFERENCES}

1 Simms I, Stephenson JM. Pelvic inflammatory disease epidemiology: what do we know and what do we need to know? Sex Transm Infect 2000;76:80-7.

2 Østergaard L, Andersen B, Møller JK, et al. Home sampling versus conventional swab sampling for screening of Chlamydia trachomatis in women: a cluster-randomized 1-year follow-up study. Clin Infect Dis 2000:31:951-7.

3 Scholes D, Stergachis A, Heidrich FE, et al. Prevention of pelvic inflammatory disease by screening for cervical chlamydia infection. N Engl J Med 1996;334:1362-6.

4 Berger RE, Alexander ER, Monda GD, et al. Chlamydia trachomatis as a cause of acute "idiopathic" epididymitis. N Engl J Med 1978;298:301-4.

5 Furuya R, Takahashi S, Furuya $S$, et al. Is seminal vesiculitis a discrete disease entity? Clinical and microbiological study of seminal vesiculitis in patients with acute epididymitis. J Urol 2004;171:1550-3.

6 Chen MY, Donovan B. Genital Chlamydia trachomatis infection in Australia: epidemiology and clinical implications. Sexual Health 2004;1:189-96.

7 National Centre in HIV Epidemiology and Clinical Research. Annual surveillance reports. Available at www.med.unsw.edu.au/nchecr/, accessed 24 July, 2004.

8 Chen MY, Fairley CK, Donovan B. Nowhere near the point of diminishing returns: correlations between chlamydia testing and notification rates in New South Wales. Aust N Z J Public Health 2005;29:249-53.

9 Hocking J, Fairley C, Counahan M, et al. The pattern of notification and testing for genital Chlamydia trachomatis infection in Victoria, 1998-2000: an ecological analysis. Aust N Z J Public Health 2003;27:405-8.

10 McAnulty J, Delpech V, Habib M. Notifiable sexually transmitted diseases, New South Wales, 1991-1999. NSW Public Health Bull 2001;12:16-18.

11 Public Health Division. The health of the people of New South Wales: report of the Chief Health Officer. Sydney: New South Wales Health Department, 2000.

12 Muscatello D, Travis S. Using the International Classification of Diseases with HOIST. NSW Public Health Bull 2001;12:289-93.

13 SAS [computer program]. Version 8.2. Cary, NC: SAS Institute Inc, 2001.

14 National Centre for Classification in Health. The International Statistical Classification of Diseases and Related Health Problems, 10th Revision, Australian Modification (ICD-10-AM). Sydney: University of Sydney, 2002.

15 Australian Bureau of Statistics. Australian historical population statistics. Canberra: ABS, 2003.

16 Weström L, Eschenbach D. Pelvic inflammatory disease. In: Holmes K, Mardh P, Sparling $P$, et al. Sexually transmitted diseases.3rd ed. New York: McGraw-Hill, 1999.

17 Rolfs RT, Galaid El, Zaida AA. Pelvic inflammatory disease: trends in hospitalizations and office visits, 1979 through 1988. Am J Obstet Gynecol 1992; 166:983-90.

18 Kamwendo F, Forslin L, Bodin L. Decreasing incidences of gonorrhoea and chlamydia associated acute pelvic inflammatory disease. Sex Transm Dis 1996;23:384-91

19 Kurtz R, Doherty J. Hospitalizations for pelvic inflammatory disease in Canada 1983/84-1993/94. Canada Communicable Diseases Report 1998; $24: 1-5$

20 Egger M, Low N, Smith GD, et al. Screening for chlamydial infections and the risk of ectopic pregnancy in a county in Sweden: ecological analysis. BMJ 1998;316:1776-80.

21 Ducket SJ. Australian hospital services: an overview. Australian Health Review 2002;25:2-18. 
22 Chen MY, Pan Y, Britt $\mathrm{H}$, et al. Trends in clinical encounters for pelvic inflammatory disease and epididymitis in a national sample of Australian general practices. (submitted).

23 Grulich $A E$, de Visser RO, Smith AMA, et al. Sexually transmissible infection and blood-borne virus history in a representative sample of adults. Aust N Z J Public Health 2003;27:234-41.

24 Bowden FJ, Jacups S, Huffam S, et al. Azithromycin and pelvic inflammatory disease in the Northern Territory [letter]. Med J Aust $2001 ; 174: 366-7$
25 Wølner-Hanssen P, Eschenbach DA, Paavonen J, et al. Decreased risk of symptomatic chlamydial pelvic inflammatory disease associated with oral contraceptive use. JAMA 1990;263:54-9.

26 Weisberg $E$, Fraser IS, Goss S. The decline in popularity of the intrauterine device. A survey of general practitioner attitudes and practices in New South Wales. Med J Aust 1994; 160:19-21.

27 Chen MY, Donovan B. Changes in testing methods for genital Chlamydia trachomatis in New South Wales, Australia, 1999 to 2002. Sexual Health (accepted). 